\title{
How to Think Straight About Psychometrics:
}

\section{Improving Measurement by Identifying its Assumptions} Víthor Rosa Franco ${ }^{1}$; Jacob Arie Laros ${ }^{2}$; Marie Wiberg ${ }^{3}$; and Rafael Valdece Sousa
Bastos $^{1}$

\footnotetext{
${ }^{1}$ São Francisco University, Campinas, São Paulo, Brazil

${ }^{2}$ University of Brasília, Brasília, Distrito Federal, Brazil

${ }^{2}$ Umeå University, Umeå, Västerbottens län, Sweden
}

\begin{abstract}
The aim of the current study is to present three assumptions common to psychometric theory and psychometric practice, and to show how alternatives to traditional psychometrical approaches can be used to improve psychological measurement. These alternatives are developed by adapting each of these three assumptions. The assumption of structural validity relates to the implementation of mathematical models. The process assumption which is underlying process generates the observed data. The construct assumption implies that the observed data on its own do does not constitute a measurement, but the latent variable that originates the observed data. Nonparametric item response modeling and cognitive psychometric modeling are presented as alternatives for relaxing the first two assumptions, respectively. Network psychometrics is the alternative for relaxing the third assumption. Final remarks sum up the most important conclusions of the study.
\end{abstract}

Keywords: Psychological measurement; item response theory; network psychometrics; psychometrics. 


\section{How to Think Straight About Psychometrics:}

\section{Improving Measurement by Identifying its Assumptions}

Is it possible to measure psychological entities? This question, albeit less troublesome for most current psychology researchers (Borsboom, 2005; Stanovich, 2012) were a main concern for scientists in the beginning of the $X X^{\text {th }}$ century. Campbell (1928) and others argued bluntly that psychological entities cannot be properly concatenated. Therefore, measurement in psychology must be impossible and scientific psychology as well. This, of course, was not well received by most psychologists at the time (Hull, 1943). One of the most influential theories on psychological measurement from this period was Stevens' (1946) operational view on measurement. This theory popularized the measurement levels, which allowed psychologists to define their variables as a different type of measures than those from hard sciences.

An older field, known as psychometrics, was being developed since the beginning of the same century (Jones \& Thissen, 2006). From the classical test theory to the item response theory, several models to measure psychological constructs were developed (van der Linden \& Hambleton, 2013), allowing for measurement instruments to be constructed as well (Furr, 2011). This development was not without controversy. Trendler (2009), for instance, says that measurement in psychology, as defined by psychometric theory, is not scientific. Michell (1997) agrees in some degree, stating that psychometrical methods do not allow for true quantitative measures to be attained. A more balanced view is sustained by Sijtsma (2012), who affirms that the two measurement approaches proposed to psychology—the statistical (i.e., the psychometrical approach) and the physical (i.e., measurement theory)—can be useful. However, they are usually not as useful as they could be, as they disregard meaningful psychological theory. 
The aim of the current study is to present three assumptions common to psychometric theory and practice. Focusing mainly on the statistical approach to measurement, we also present how alternatives to traditional psychometrical approaches can be used to improve measurement in psychology. The rest of this paper is structured as follows. In the next section, we explain how quantitative and qualitative reasoning impacts theorizing in psychology, originating the latent framework in psychometrics. We then present the latent framework as the basis for the three most popular theories in psychometrics and list the three assumptions common to all these theories. The next three sections discuss each of the three assumptions, presenting how research in psychological measurement can better explore each of these assumptions. The paper ends with a number of concluding remarks.

\section{Quantitative and qualitative thinking in psychology}

To assure a scientific status, researchers in the field of psychology have preferred to use quantitative practices for data analysis (Mertens, 2014). This happened because, in the beginning of the $\mathrm{XX}^{\text {th }}$ century, to be considered a science, any field of study should rely on mathematics and formal logic (Price, 1986). Nevertheless, the theorization in psychology is still, and increasingly (Myung \& Pitt, 2001; Towsend, 2008), done on basis of natural language, meaning that relations between variables are not objectively defined. This, on itself, is not a problem, given that qualitative thinking can be beneficial for science. However, methods and theorization should suit the research question, not the other way around.

In methodological textbooks for undergraduate and graduate students (e.g., Shaughnessy et al., 2014), much is said about how methods and data analysis should properly be selected to answer each type of research question. For instance, Kish (2004) 
proposes that every research can be of one - or a combination of - design category: realistic; representative; and randomized. Realistic research designs are those centered on being profound about a singular subject, usually using qualitative research techniques and are meant, mainly, to be of a descriptive nature. Representative research designs should be used when one wants to know if a characteristic is generalizable to a population, as in survey research. Finally, randomization designs are, basically, experiments: "randomization" is used to express the random group assignment and are defined as the type of design that should be used to infer causal relations.

Despite all the different types of methodological designs that exist, they only help to answer an already posed research question. The problem for the development of psychological science is, therefore, not only dependent on the research design, but also on how the research question was posed (Shaughnessy et al., 2014). This is a considerably less discussed topic in scientific psychological literature. Most textbooks and tutorial papers will focus mainly on where research questions come from, rather than the procedures used to derive them (e.g., Sandberg \& Alvesson, 2011). For instance, Shaughnessy et al. (2014) suggests there are two important sources for scientific theorization: past research or our personal experiences. Provided that both are further and critically evaluated, using a proper method, they are valid sources for theorizing. However, this does not answer the question of what theorizing is and how to properly do it. For instance, given a prior scientific result, how does one create new hypotheses or proposes modifications to a given theory?

Theorizing is certainly not an effortless endeavor (Thabane et al., 2009), and defining what is proper theorizing is not a straightforward effort. However, some authors propose some alternatives. As is usual in science, there is no unique way for theorizing, but it can be categorized in, at least, two types, depending on the amount of 
formalization used to describe the phenomena of interest (Myung \& Pitt, 2001).

Formalization is used here to define the use of mathematical, logical or any objective language (Shoenfield, 2018) in contrast to the natural language, such as English, Portuguese, Swedish, and others (Manning et al., 1999). Therefore, the first type of theorizing, which is also the most common in psychology and other sciences (Townsend, 2008), is the natural language theorization. As the name suggests, this type of theorization is done by simply stating, in natural language, what, how and why empirical data is how it is. It also involves a lot of rationalization over past empirical results. A simple example can be given by the theory of cognitive dissonance, classically defined as the mental discomfort experienced by a person who simultaneously holds two or more contradictory beliefs, ideas, or values (Festinger, 1962). This definition, despite being clear and meaningful for most individuals who understand the English language, does not explain, for instance, how mental discomfort is caused by holding contradictory beliefs. Most psychological theories and hypothesis have this format. They vaguely state some expected relation between variables, without acknowledging the process that originates this expected relation.

Even theories and hypothesis that are more preoccupied with the process and with more complex relations between variables do it by using natural language, meaning they will, for the nature of natural languages, lack precision. For instance, the multicomponent model of working memory by Baddeley and Hitch (1974) states that three components are necessary for working memory: the central executive, the phonological loop, and the visuospatial sketchpad with the central executive functioning. For the current presentation, it is not necessary to describe these components. It is necessary only to know that the authors stated that all the components are necessary to prevent decay of relevant memory information (Baddeley \& Hitch, 
1974). However, they do not explain how the decaying process works. When does it begin? Is the decaying rate constant or variable? Does the decaying process have some limit of "data exclusion" or the information can be completely lost? For illustrative purposes, Figure 1 shows four possible decaying rate models that could all be true, given the definition used by the authors.

\section{Figure 1}

Four possible models for decaying rate in memory tasks.

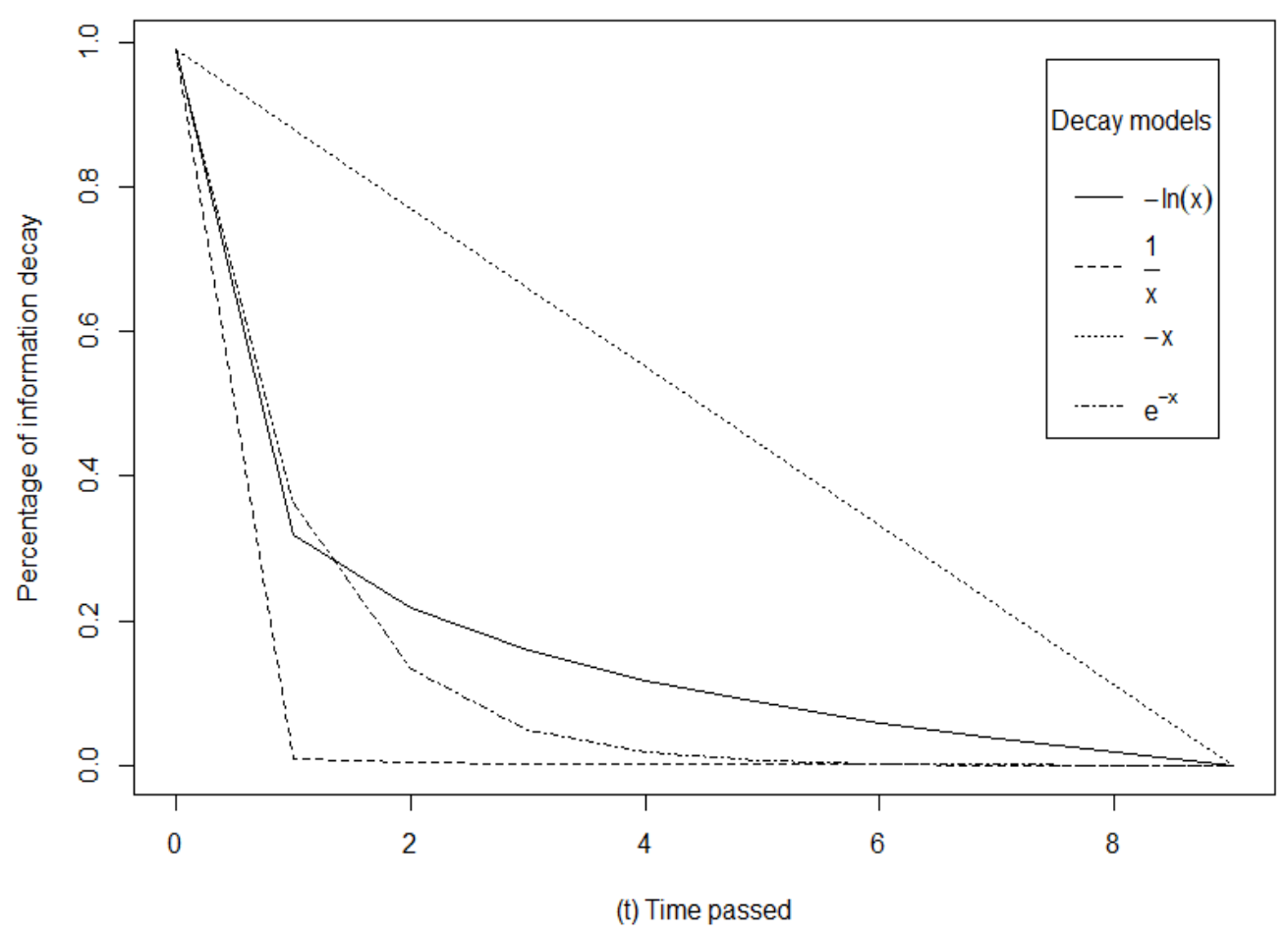

The exposition so far does not allow to imply that natural language does not have its place in scientific theorization. The true message is that when describing quantities and patterns, a more appropriate symbolical tool should be used; which is mathematics. This has been the practice of what is known as mathematical psychology (Coombs et al., 1970): an approach to psychological research that is based on mathematical modeling and on the establishment of psychological rules of quantifiable 
psychological processes. Again, psychology researchers seem to prefer to use quantitative practices for data analysis, but quantitative reasoning and quantitative theorizing seems still to be lacking (Towsend, 2008). One example of use of quantitative theorizing is the latent variable theory on psychometrics (McDonald, 2013).

\section{Psychometrics and its three assumptions}

Psychometrics is a field in psychology concerned with the theory and technique of psychological measurement (McDonald, 2013). Three major theories were developed to explain the relations between observed data and psychological traits: classical test theory (CTT); common factor theory (CFT); and item response theory (IRT). Even though many authors defend, for instance, that IRT is superior to CTT (Borsboom, 2005; Hays et al., 2000; Reise et al., 2005), all these psychometric theories can be understood as different applications of the same general Latent Variable Theory (LVT; McDonald, 2013). LVT holds that psychological variables (or constructs) are explanatory variables which are not directly observable, but inferred from their effects on human behavior.

The following equations, that can respectively be used to express CTT (1), CFT (2), and IRT (3), clarify why all these theories are related to LVT:

$$
\begin{gathered}
X=T+\varepsilon, \\
X=\lambda T+\varepsilon, \\
g(X)=f(T),
\end{gathered}
$$

where $X$ stands for the observables, $T$ for the true, latent, score, $\lambda$ for the factor loadings (or factor weights) and $\varepsilon$ for the random error. All equations can be understood as an extension of the previous, with the same monotonic relation between observed and latent variables. The functions $g(X)$ and $f(T)$ are usually represented, respectively, by 
probability mass functions (e.g., binomial or categorical distributions) and link functions (e.g., logistic function or cumulative normal function) to properly scale the observed and latent variables.

It is possible to perceive that all functions generate similar inferences about relations of true and observed scores. The differences in the results rely, mostly, on the methods used to estimate the parameters of these equations (McDonald, 2013), meaning that, from a more computational perspective, these methods are rather different. The true score in Equation (1) is normally estimated using sum or average scores, Equation (2) is tested by means of confirmatory or exploratory factor analysis (Thompson, 2004), and Equation (3) will be tested by some item response model (IRM; van der Linden \& Hambleton, 2013). All these methods harness some pragmatic assumptions, being some of them testable (e.g., unidimensionality assumption; Stout, 1987), but some others are untestable (Michell, 2000). For instance, some variation of the logistic function is generally assumed as the item response function (IRF) for $f(T)$ in Equation (3). Regardless of being usually taken as an obvious assumption, due to a traditional psychophysical empirical finding on tone and loudness perception (Fechner, 1860), there is no direct test for the validity of this assumption. This is to say that, given that the true score is latent and not directly experimentally controllable, it is impossible to test if the logistic function is really the function that relates latent to observed variables (Levine, 2003). However, it is possible to test if the observed IRF is monotonic in relation to the latent IRF (Junker \& Sijtsma, 2000).

At this point it should be noted that, despite harsh, this criticism does not necessarily invalidate the general LVT approach for psychometrics. This general psychometric approach can be defined as a statistical approach to measurement (Sijtsma, 2012). A statistical approach is characterized by accepting the assumptions of 
some statistical or mathematical measurement model, using them to establish "quality" thresholds on data. For instance, when using Factor Analysis, items with low factor loadings are usually suggested to be discarded (Thompson, 2004). On the other hand, factor analysis is based upon linear regression, meaning that, if the true process is quadratic or relies on any other non-monotonic function, the estimates of factor loadings are probably biased (McDonald, 1965). For problems like that, a second approach for dealing with psychological data could be the physical approach (Sijtsma, 2012). The physical approach consists of testing if a particular mathematical structure is true for the data. For instance, utility theory uses a number of axioms to define rational behavior (von Neumann \& Morgenstern, 1944). However, people not always behave rationally, meaning these models will not always properly fit the data (Allais, 1953). The usual interpretation is that people are not rational. Kahneman and Tversky (1979), on the other hand, proposed the prospect theory, changing the assumptions of the utility theory, with a consequence to changing the interpretation on humans' decision-making behavior.

The statistical and physical approaches to measurement provide researchers with two different mindsets, respectively: rejecting the data if the model has a poor fit; or rejecting the model if it has a poor fit to data. None is, for itself, the best approach for proper inferences. Sometimes it is better to reject the data after a low fit of the model, given that there may be some bias on the data collecting process (Shaughnessy et al., 2014). On the other hand, if data are properly collected and the model used for testing it systematically shows a pattern of bad fit, maybe an alternative model should be tested. However, most researchers are not even aware of the existence of the physical approach to measurement (Michell, 2017), as it demands more knowledge on mathematics and 
experimental design. Both requirements make research and developing quality measurements more difficult for several areas in psychology.

Three major critiques can then be elaborated about the traditional psychometric practice. Each critique is related to one of three assumptions regarding psychological measurement, as we derived from the LVT and the statistical approach to measurement. First, psychological measurement is based on using pre-conceived models that, sometimes, are non-testable and have higher priority than the empirical data. We call this the structural validity assumption. Second, psychological measurement is based on models that, sometimes, do not mirror the psychological phenomena or processes they are intended to represent. We call this the process assumption. Finally, traditional psychological measurement depends heavily on constructs, which are not observable and can be sometimes difficult to define; therefore, difficult to give a proper operationalization. We call this the construct assumption. These assumptions do not need to have a specific hierarchy of complexity or necessity.

As with several other problems within science, relaxation or thorough testing of the assumptions can help science to improve (Kanazawa, 1998). Nonparametric Item Response Modeling (NIRM; Sijtsma \& Molenaar, 2002) can be used to test or to relax the structural validity assumption, but it still relies on the same process and construct assumptions from Parametric Item Response Theory. Cognitive Psychometric Modeling (Embretson, 2010) can be used to test or relax both structural validity and process assumptions, but is still relying on the construct assumption. Finally, network modeling (Epskamp \& Fried, 2018), based on the statistical approach can be used to relax all three assumptions, while, of course, depending on a different set of assumptions.

\section{Structural validity assumption and Nonparametric Item Response Modeling}


The structural validity assumption is the exact mathematical implementation of psychometric models. For instance, traditional factor analysis assumes latent variables to be linearly related to observed variables (Thompson, 2004). The error of measurement has an expectation of zero and, for adequate fitting, a normal distribution is generally used for modeling the error. The structural validity assumptions can be thought of as being the least related to a particular psychological theory, but the most related with the statistical, mathematical, or computational feasibility of the implementation of a model (e.g., Griffith \& Akio, 1995). A good historical example of changing a structural validity assumption occurred in the case of the transition between initial IRMs and the logistic and Rasch models. Initially, the normal cumulative density function was used as the IRF for the binary IRMs (Lord, 1953). Nevertheless, at the time of the development of the first IRMs, computing this IRF was computationally extensive. For this reason, some authors proposed changing from the normal to the logistic cumulative density function as the IRF for IRMs (Birnbaum, 1968). Mathematically, and for modern computers, this difference makes little to no difference, but at the time it was necessary so using IRMs was feasible (Rasch, 1960).

Apart from changing mathematical characteristic of the models, most of the structural assumptions in IRMs can also be relaxed using NIRMs (Sijtsma \& Molenaar, 2002). NIRM is not only a different class of item response models, but also a whole different approach to modeling response patterns. For both NIRM and Parametric IRMs, there are three main assumptions about the relations between the observed scores and the latent trait. All these assumptions are specificities of our structural validity assumption. The first is that of unidimensionality, which simply means that the observed scores have only a single latent cause (Stout, 1987) — or a single more relevant cause. 
While there is much theoretical support for multidimensionality in psychological measurements (Knol \& Berger, 1991; Reckase, 2009), given the complexity of psychological phenomena, many authors defend that unidimensional measurements should be preferred (Nunnally, 1978; Sijtsma \& Molenaar, 2002). The main argument standing the latter can be clearer stated with an analogy. If you would use a scale to measure your weight and your height at the same time, what a score of 104 would mean? Supposing there was no standard unit for both measures, this score would be meaningless to making conclusions about those magnitudes apart. Therefore, multidimensional scores, despite being probably more representative of psychological phenomena, should be avoided so meaningful measures can be achieved (Heene et al., 2016).

The second assumption is that of local independence, which states that the observed score of individual $i$ on item $k$ does not depend on the response he gave in any other $j$ item, conditioned on the latent trait (Zhang \& Stout, 1999). Finally, the third assumption is monotonicity, which states that the probability of getting an item right (or of endorsing a higher score in a Likert scale) augments with increasing latent trait (Junker \& Sijtsma, 2000). Another assumption, not common to all IRMs, however, is that of nonintersecting item response functions (IRFs; Rosenbaum, 1987). This assumption is used, for instance, by the Rasch Model and by the One-Parameter Logistic Model (1PLM; van der Linden \& Hambleton, 2013). For ordering items by their difficulties' estimates, these assumptions need to hold, simply because when IRFs intersect there is an interaction effect between individuals' levels of the latent trait and items difficulties (Sijtsma \& Molenaar, 2002). This means that the ordering of item difficulty is not the same for all individuals, but depends on their latent traits. Obviously, this is not a desirable property when you want to create a standard test. 
NIRM begins to differ from parametric IRM when the operationalization of these assumptions takes place. For instance, a common monotonic function relating the latent trait with the observed scores is the logistic function:

$$
\operatorname{Pr}(X=x \mid \theta, \delta)=\frac{e^{\theta-\delta}}{1+e^{\theta-\delta}}
$$

where $\theta$ stands for the level of the individual's latent trait and $\delta$ for the level of item's difficulty. All the lines depicted in Figure 2 are representations of this function, with different values for the subtraction $\theta-\delta$.

\section{Figure 2}

Depictions of logistic functions.

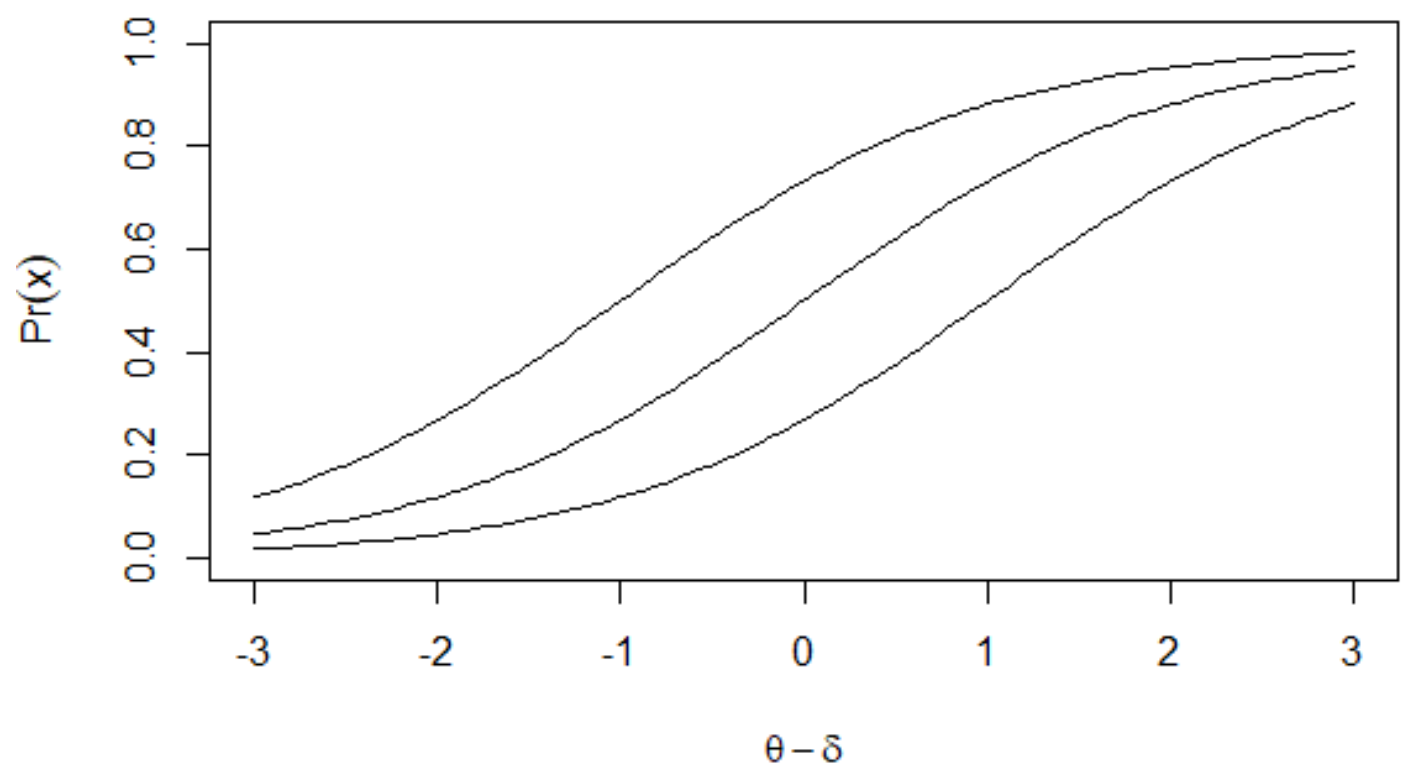

It is possible to see that the IRFs will always be "S" shaped. For NIRMs, however, any function can be used, since it does not disregard the monotonicity. The general formulation of IRFs for NIRMs is the following

$$
P_{i}\left(\theta_{a}\right) \leq P_{i}\left(\theta_{b}\right)
$$


which implies that, provided that the function is a nondecreasing function of $\theta$, any function can be used to relate the latent trait with the observed response. Figure 3 depicts five different functions, all which can be used as IRFs in a NIRM perspective.

\section{Figure 3}

Depictions of valid functions IRFs in a NIRM perspective.

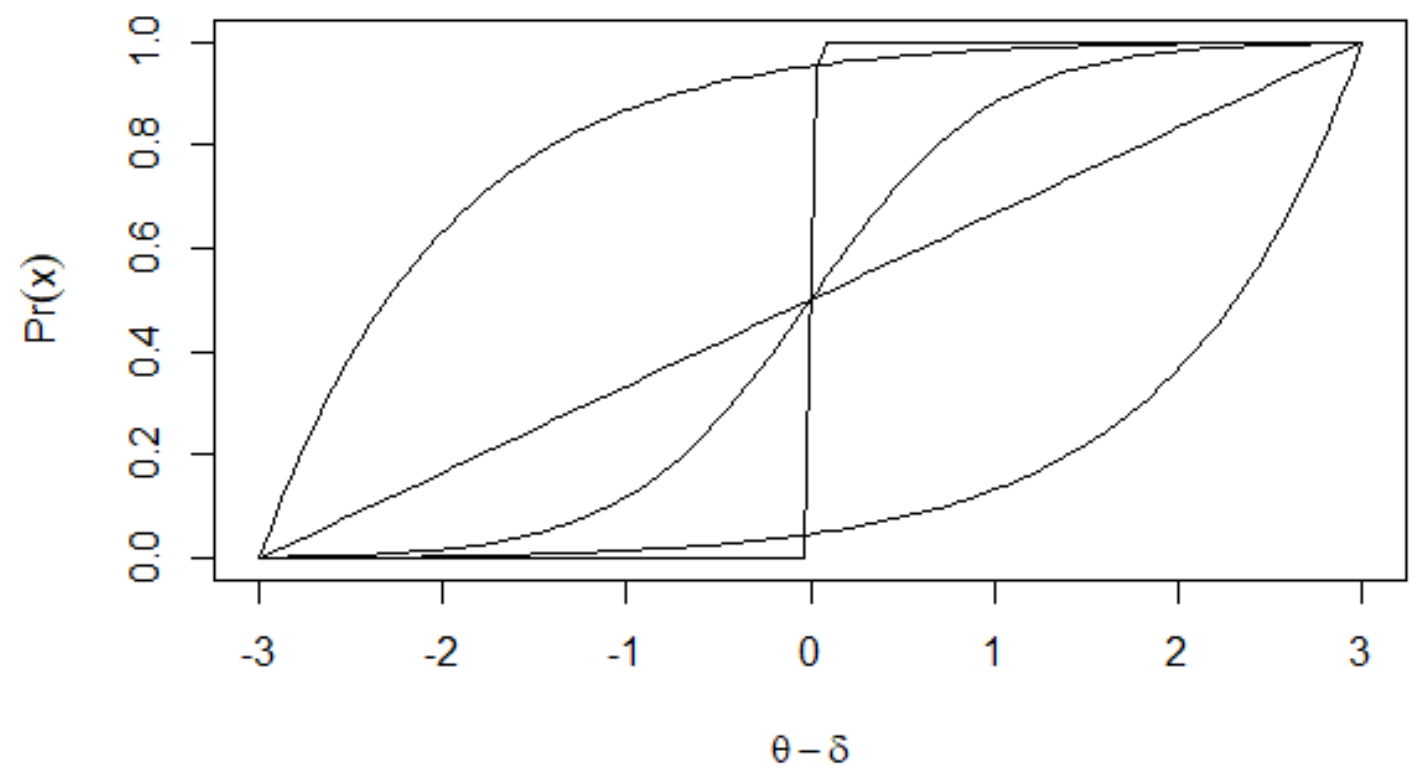

As there are, in principle, an infinite number of functions that can conform to the less restrictive forms of NIRMs, it would be computationally extensive (or impossible) to test, for instance, exactly what function the IRF follows (Ferraty \& Vieu, 2006). Therefore, in a NIRM perspective, instead of making complex estimates of latent variables, the fit of the model is given by the capacity of the data to follow the relaxed assumptions. This means that, for example, the data could give a result like that in Figure 3, when using NIRM, but, if fitted with a 1-PLM, could result in what was shown in Figure 2. This would probably happen because while NIRMs will test the assumptions it makes, parametric IRMs forces data into its functional form. 
Between the most well know models of NIRMs are those from Mokken Scale Analysis (MSA; Mokken, 1971). A Mokken scale is a non-parametric, probabilistic version of the Guttman scale (Mokken \& Lewis, 1982). Both Guttman and Mokken scaling assumes that items have a hierarchical order, meaning that respondents who answered a difficult question correctly should also answer an easy question correctly. The main difference between Guttman and Mokken scales is that Guttman scaling assumes that respondents who answered a difficult question correctly will necessarily answer an easier question correctly. When that does not happen, it is said to be a Guttman error (Meijer, 1994). Nevertheless, in real evaluation scenarios usually people do not respond deterministically, but accordingly with some stochastic process, better modeled with MSA or a parametric IRM (PIRM).

It is important to note that there are many other non-parametric and semiparametric models that can be found on the literature. Semi-parametric models are the ones that alleviate just some, not all, of the assumptions made by parametric models (Dey et al., 2000). Many Bayesian models of such kind have been created (e.g., Miyazaki \& Hoshino, 2009; Wang et al., 2013). There are also tests and models for inferential analysis that are centered on testing the common assumptions for PIRMs (e.g., Stout, 1987; Straat et al., 2013). All these different techniques make it possible to test and model a larger range of items, without being needed to limit test and scale construction to what is only permitted by traditional IRMs.

However, all those different models share an important limitation, as stated by Sijtsma (2012). Using those techniques, the measurement is dependent on the questionnaire - or test, or scale — data, meaning that they all assume that the observed scores are caused by a generic latent variable related to the items and to the respondents. This structural validity assumption makes it easier to mistake the prescriptive structure 
of a statistical measurement model with the theoretical structure of the attribute of interest. Nevertheless, meaningful measurement is possible only if enough is known about the attribute so as to justify its logical operationalization into prescriptions from which a measurement instrument can be developed (Michell, 2017; Sijtsma, 2012; Trendler, 2009). Despite theories about attributes in psychology often not being precise enough to justify a logical operationalization, the emerging field of cognitive psychometric modeling has been presented as an interesting alternative (Embretson, 2010).

\section{Process assumption and Cognitive Psychometric Modeling}

The process assumption is the definition on how basic constructs, or latent variables, relate to each other to compose a particular psychological model or theory. For instance, the IRT assumes that the probability of correct answering a question is a function of the respondent's latent trait and the difficulty of the items. The signal detection theory, on the other hand, assumes that the probability of correct answering a question is a function of the criterion and discrimination of the respondent (Stanislaw \& Todorov, 1999). In this case, it is possible to distinguish the measurement made by these theories as, for the signal detection theory, it makes a difference if correct responses were a hit or a correct rejection, and if the incorrect responses were a false alarm or a miss. For IRT, it usually matters only if the response was correct or incorrect. This entails in the fact that both theories make different assumptions on the underlying process controlling response patterns on the proposed quantitative model.

A quantitative model is a representation of a phenomenon using techniques and procedures due to mathematics and statistics (Edwards \& Hamson, 2007). Therefore, a cognitive model is a representation of cognitive phenomena using the same class of 
mathematical and statistical techniques and procedures (Lee \& Wagenmakers, 2014). Lewandowsky and Farrell (2010) describe three different classes of quantitative models. The first class contains models of data description. As the name suggests, they only describe relations between variables. They are explicitly devoid of psychological content, although the modeled function constrains possible psychological mechanism to the phenomena. An example is linear regression models (Faraway, 2016). The second class is the one of process characterization. These models postulate and measure distinct cognitive components. Yet, they are neutral about how specific instantiations underpinning the cognitive components work. An example is the multinomial processing trees model (Erdfelder et al., 2009). Finally, the third class is the one with models of process explanation. Like characterization models, their advantage stands on hypothetical cognitive constructs. However, they provide detailed explanation about those constructs and how are they related. An example is the generalized context model (Nosofsky, 1986).

Traditional psychometric models can be thought as descriptive models, given that they can be described themselves as only linear or generalized linear regressions with latent predictors (Bock, 1997). Considering psychometric models from this perspective enables to perceive that, despite being important tools to psychological research, traditional IRMs lack explanatory meaning. Therefore, several aspects of psychological phenomena are not taken into account. Sijtsma (2012) states that IRT leaves psychology out of the equation when proposing psychological models of measurement, resulting in fruitless insights for psychological phenomena. However, it is important to note that it is not the use of a statistical framework based on latent variables that is the strongest limitation of IRT. The strongest limitation is to use exclusively descriptive models (i.e., 
a strong process validity assumption) for developing measurement models, which have no concern for the processes that generated the observed data structure.

The cognitive modeling approach can then be used to enrich IRMs and give more significance to the measurement process in psychology. Despite not formally defined as such, the Cognitive Psychometric Models (CPM) approach has been used as a good alternative for traditional IRMs (Embretson, 2010). One prominent CPM is Tree Based IRMs (TIRMs; LaHuis et al., 2018). This kind of model helps to understand in which order latent variables influence each other to cause the observed response patterns. Empirical comparison of different TIRMs can provide proper evidence of validity for a measure, when compared, for instance, with simply correlating expected-to-be-related measures (Borsboom et al., 2004). Figure 4 illustrates two hypothetical competing TIRMs for the measurement of personality data. Both models state that people have different propensities to act aggressively or peacefully. However, the model on the left states that the probability of a respondent giving an aggressive or a peaceful response depends only on the parameter $\alpha$, which can be thought as his propensity to act in a given way (e.g., his disposition). On the other hand, the model on the right decomposes the process, stating that self-control, measured by the parameter $\beta$, has an important role on regulating individual's actions. Traditional analysis in psychometrics and psychology would only test correlations between these measures (e.g., Kim et al., 2008), providing only descriptive relations for the constructs. The use of the TIRMS makes it possible to conclude what process is more likely to have originated the data at hand. In the example, individuals with more peaceful personality do not need to have self-control, given that they simply act as is socially expected. This kind of conclusion would be difficult, or even impossible, to be drawn by traditional psychometrical analysis. 


\section{Figure 4}

Two hypothetical competing TIRMs for the measurement of personality data related to observed behavior.

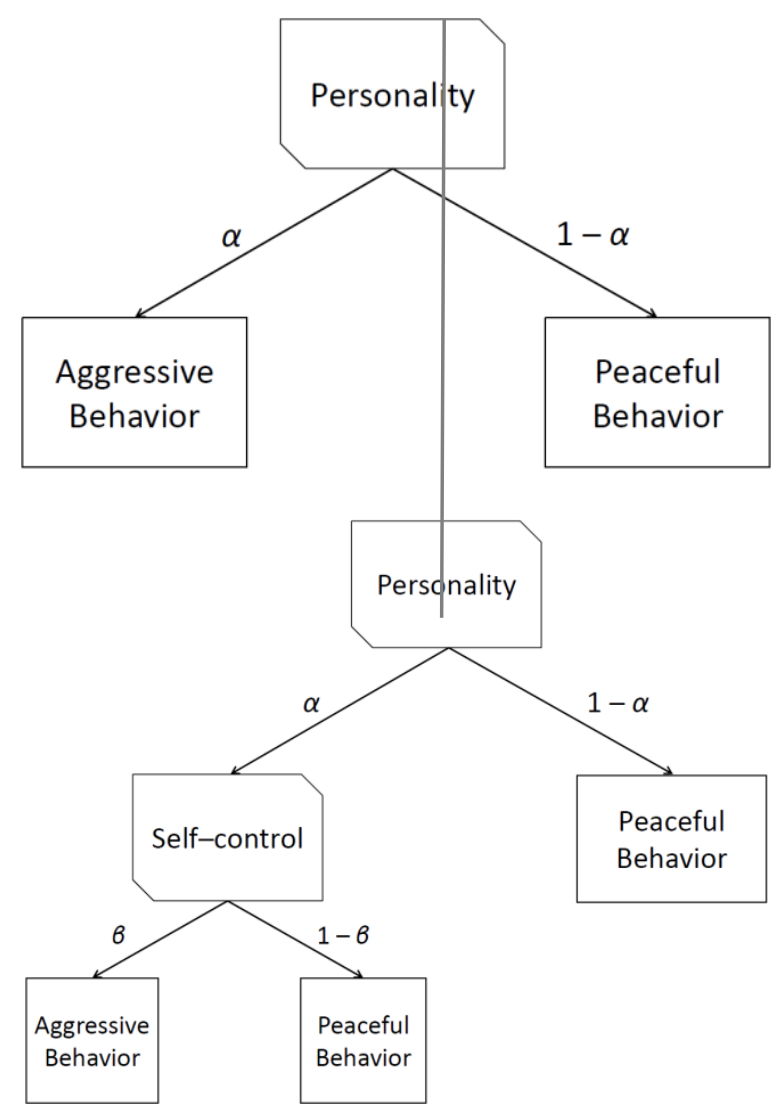

Following what is proposed by Sijtsma (2012), a measurement occurs only when the relations between the observed measure and the attribute of interest are logically and formally established, making it possible to operationalize the phenomena of interest. Characterization and explanation's CPMs can be used for this end, as illustrated by the example of Figure 4. Certainly, on the other hand, for the proper use of CPMs one cannot only rely on questionnaire or test data. This can be a difficult task. Kagan (2005) criticized the common psychometric practice on relying almost exclusively on the semantic structures activated when participants give their responses to questionnaires. 
The author also proposes the use of alternative data sources, such as motor activity, distress to unfamiliar visual, auditory, and olfactory stimuli, and others. Sijtsma (2012) states that these alternative data types are rarely used in psychometrical research. Cognitive psychology researchers who use cognitive modeling approaches, on the other hand, are more used with searching for different data sources for their models (Lee, 2011).

One way of increasing the number of data sources and, therefore, making a more robust and valid measurement model, is collecting data in experimental sets (Sijtsma, 2012). This is a good approach for two main nested reasons. First, because using experimental sets reduce the noise and random variance in the data (Kish, 2004). As many sources of confounding effects are controlled in experiments, each new relevant measurement reduces the amount of unexplained variance. The repeated measurement design is the design that reduces the noise the most (Cook et al., 2002). The second reason, which is nested in the first, is because experimental sets in psychology usually control for the external influences in individual's behavior. Therefore, the variance that is left can be due only to noise or individual differences (Bacon, 2004).

Using CPMs and experimental sets are still a novel approach to psychological measurement, which has the potential to approximate the meaning of measurement in psychology with that of physics (Sijtsma, 2012). Nevertheless, for some authors (e.g., Gould, 1996), measurement in psychology will always be impossible, given that IRMs, and even CPMs, define measurement as the estimates for some latent variable. This means that, for some authors, it is not enough to relax or test the structural and process validity assumptions, but is also necessary to directly observe the measured property or feature. Despite this being problematic for psychology as a whole (Borsboomet al., 
2003; Trendler, 2013), measurement in fact does not need to be defined in terms of latent variables. Network psychometric modelling can be used to this end.

\section{Construct assumption and Network Psychometrics}

The construct assumption is the definition of a measure as the latent variable that explains the variance of observed variables. All models presented so far rely on this assumption, as the estimates of the magnitude of the latent variables are of central interest, and not the observed variables per se (Borsboom, 2005). This is an old trend in mainstream psychology to attribute mentalist causes to human behavior (Stanovich, 2012). Despite the success of this approach in many areas in psychology (Sijtsma, 2012), philosophical and mathematical critics are made to accept something that cannot be assessed or, if ever, only indirectly assessed (Michell, 1990; 1997; 2005; 2008). The main alternative for the construct-based approached to psychological measurement is found in the form of network psychometric modeling (Constantini et al., 2019).

Probabilistic graphical modeling (Lauritzen, 1996) is a statistical approach, derived from the mathematical graph theory, used to model multivariate conditional dependencies between variables. In this sense, factor analysis and item response models can be considered as special cases of probabilistic graphical models, where dependencies between observed variables are conditioned on latent variables (Kruis \& Maris, 2016). For the probabilistic graphical models proposed by Lauritzen (1996), however, no latent variables are considered. Instead, dependencies between any two observed variables are explained by their relations to a third observed variable. One of such models is the partial correlation graphical model, also known as partial correlation network model (Epskamp \& Fried, 2018). This model has been of growing interest in the field of psychometric construct analysis, such as mental health related ones 
(Borsboom, 2017), where the existence of a common latent cause is a controversial issue.

The probabilistic graphical modelling approach to psychometrics, also named network psychometrics (Epskamp et al., 2017), is considered as a part of the statistical approach to measurement because it is not particularly interested in the measurement level of the observed variables. For instance, partial correlations can be calculated from polychoric correlations, combined with regularized regressions, if the observed variables are ordinal (Golino \& Epskamp, 2017). It is generally not the objective to estimate interval or ratio measures to predict observed variables, nor are the values of the observed variables transformed to interval or ratio measures. However, some network psychometric models, such as the network Ising model, have been shown to be equivalent to traditional latent common cause models, such as factor analytical models and item response models (Marsman et al., 2018). This means that not always network psychometrics will present a true alternative to traditional psychometrics (in terms of the construct assumption), only when it avoids latent variables (Kruis \& Maris, 2016; Lauritzen, 1996).

One good example of using network psychometrics, and abandoning latent variables to measure psychological entities, is the model of general intelligence proposed by van der Maas et al. (2006), known as the mutualism model of intelligence. Traditionally, the study of human intelligence was concomitantly developed with the psychometric factor analytic model (Buckhalt, 2002). This is represented by the fact that one of the main discussions in the study of intelligence is not about the existence of a true latent variable, but about how many dimensions describe this assumed latent variable the best (e.g., Golino \& Demetriou, 2017). The most traditional model, and maybe one of the best corroborated, is the $g$-factor model of general intelligence 
(Canivez \& Watkins, 2010). In this model, a single general latent variable is used to explain the variance of all observed variables. Some extensions (Canivez, 2016) involve using this $g$-factor as the cause of other latent variables (named specific factors), or with other latent variables explaining residual correlations, after conditioning the $g$-factor out. These are known as the second-order and bifactor models, respectively. The traditional, second-order, and bifactor models of intelligence are respectively illustrated in Figure 5, where $g$ represents the $g$-factor, $f 1, f$, and $f n$ represent the possible specific factors, and $V 1, V \mathrm{i}$, and $V \mathrm{n}$ represent the possible sets of observed variables.

\section{Figure 5}

Graphical illustrative example of the traditional (left), second-order (middle), and bifactor (right) models of intelligence

Traditional

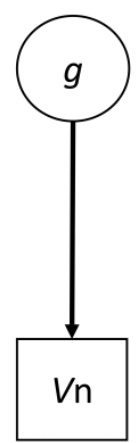

Second order

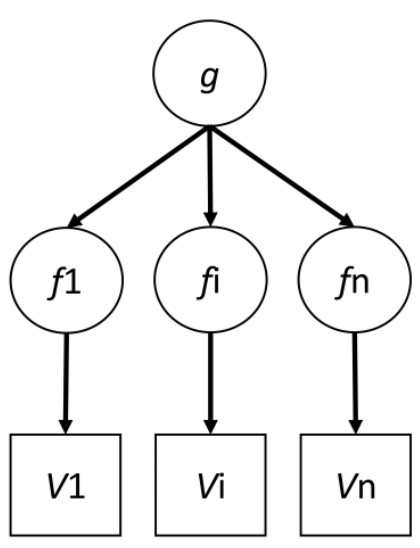

Bifactor

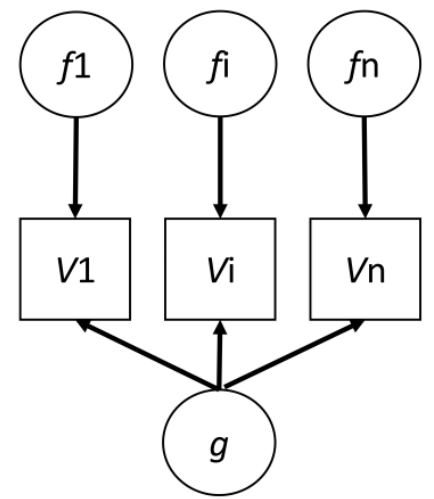

The network model proposed by van der Maas et al. (2006), on the other hand, suggests that the dynamics of intelligence are better described by a network of reciprocal causal effects. The idea of the mutualism model of general intelligence is that such reciprocal causal effects also occur during development, originating the observed correlations, for instance, on responses to items on intelligence tests (van der Maaset al., 
2017). From an empirical point of view, van der Maas et al. (2006) showed that phenomena such as the hierarchical factor structure of intelligence, the low predictability of intelligence from early childhood performance, the integration/differentiation effect, and the increase in heritability of $g$ can all be explained by the mutualism model. Despite this fact, the mutualism model has been criticized for not accounting some effects, such as distinguishing between genetic and environmental effects (Nisbett et al., 2012), that are already well studied with traditional psychometrical models. Van Der Maas et al. (2017), however, argue and present a new model that can better address most of the criticism of the mutualism model.

\section{Discussion}

The aim of the current study was to present three assumptions common to psychometric theory and practice. These are the assumption of structural validity, the process assumption, and the construct assumption. We presented the basic idea on measurement model development by adapting each of these assumptions. In a nonexhaustive manner, we also presented how alternatives to traditional psychometrical approaches can be used to improve measurement in psychology. The take home message is similar to that of Sijtsma (2012): only rigorous development of attribute theories can lead to meaningful measurement in psychology. Introducing and stressing the three assumptions in psychometrics not only allows understanding why measurement in psychometrics is as it is, but also allows to identify how to change its models so to achieve meaningful measurements.

If latent variables are acceptable and the process is assumed to be known, traditional psychometric models, such as factor analytical and item response models, can be improved by simply changing some of its mathematical functions (structural 
validity assumptions). We showed as the main examples the change between normal and logistic item response functions to the Rasch model, and the use of nonparametric item response modeling. These procedures change only minor or none of the aspects of the underlying theory about the response or cognitive/behavioral processes. In this case, developing models are mainly related to improving the fit to the data, rather than improving the theoretical description of the underlying psychological process. However, studies on validity can still be used in this approach to improve the understanding of the underlying psychological processes (Cronbach \& Meehl, 1955).

If latent variables are acceptable but the underlying psychological process is to be explored, then one should develop models that try to characterize or to explain this process (process assumption). We showed as the main examples the difference between item response theory and signal detection theory, as well as the tree item response models (as a type of characterization models). It is important to note that the signal detection theory also provides a descriptive model on respondents' response patterns, similarly to item response theory. However, differences on predictions made by both theories allow for designing studies to compare which model makes the most sense, given the experimental apparatus (Trendler, 2009).

Finally, latent variables can be abandoned and measurement in psychology will still be possible (construct assumption). This can be achieved by a statistical approach to measurement, by means of network psychometrics. The main difference between network and conventional psychometrics is that, despite the measurement level of the observed variables, the focus is to causal relations between the observed data using multivariate statistics. Depending on the objective, and the underlying theory, the researcher should decide which model/procedure to use, aiming at developing more meaningful measurements in psychology. 


\section{References}

Allais, M. (1953). Le comportement de l'homme rationnel devant le risque: Critique des postulats et axiomes de l'école américaine [The rational man's behavior in the face of risk: Critical of postulates and axioms of the American school].

Econometrica: Journal of the Econometric Society, 503-546. https://doi.org/10.2307/1907921

Bacon, D. (2004). The contributions of reliability and pretests to effective assessment. Practical Assessment, Research \& Evaluation, 9(3), 1-8. https://doi.org/10.7275/kbtm-zy59

Baddeley, A. D., \& Hitch, G. (1974). Working memory. In G. A. Bower (Ed.), The psychology of learning and motivation (pp. 47-90). New York: Academic Press.

Birnbaum, A. (1968). Some latent trait models and their use in inferring an examinee's ability. In F. M. Lord \& M. R. Novick. (Eds.), Statistical theories of mental test scores (pp. 397-479). Reading, PA: Addison-Wesley.

Bock, R. D. (1997). A brief history of item theory response. Educational Measurement: Issues and Practice, 16(4), 21-33. https://doi.org/10.1111/j.17453992.1997.tb00605.x

Borsboom, D. (2005). Measuring the mind: Conceptual issues in contemporary psychometrics. Cambridge: Cambridge University Press.

Borsboom, D. (2017). A network theory of mental disorders. World Psychiatry, 16(1), 5-13. https://doi.org/10.1002/wps.20375

Borsboom, D., Mellenbergh, G. J., \& van Heerden, J. (2003). The theoretical status of latent variables. Psychological Review, 110(2), 203-219. https://doi.org/10.1037/0033-295X.110.2.203 
Borsboom, D., Mellenbergh, G. J., \& van Heerden, J. (2004). The concept of validity. Psychological Review, 111(4), 1061-1071. https://doi.org/10.1037/0033295X.111.4.1061

Buckhalt, J. A. (2002). A short history of g: Psychometrics' most enduring and controversial construct. Learning and Individual Differences, 13(2), 101-114. https://doi.org/10.1016/S1041-6080(02)00074-2

Campbell, N. R. (1928). An account of the principles of measurement and calculation. London: Longsman, Green.

Canivez, G. L. (2016). Bifactor modeling in construct validation of multifactored tests: Implications for multidimensionality and test interpretation. In K. Schweizer \& C. DiStefano (Eds.), Principles and methods of test construction: Standards and recent advancements (pp. 247-271). Göttingen, Germany: Hogrefe.

Canivez, G. L., \& Watkins, M. W. (2010). Investigation of the factor structure of the Wechsler Adult Intelligence Scale—Fourth Edition (WAIS-IV): Exploratory and higher order factor analyses. Psychological Assessment, 22(4), 827-836. https://doi.org/10.1037/a0020429

Constantini, G., Richetin, J., Preti, E., Casini, E., Epskamp, S., \& Perugini, M. (2019). Stability and variability of personality networks. A tutorial on recent developments in network psychometrics. Personality and Individual Differences, 136, 68-78. https://doi.org/10.1016/j.paid.2017.06.011

Cook, T. D., Campbell, D. T., \& Shadish, W. (2002). Experimental and quasiexperimental designs for generalized causal inference. Boston: Houghton Mifflin. 
Coombs, C. H., Dawes, R. M., \& Tversky, A. (1970). Mathematical psychology: An elementary introduction. Englewood Cliffs, NJ: Prentice-Hall.

Cronbach, L. J., \& Meehl, P. E. (1955). Construct validity in psychological tests. Psychological Bulletin, 52(4), 281-302. https://doi.org/10.1037/h0040957

Dey, D. K., Ghosh, S. K., \& Mallick, B. K. (2000). Generalized linear models: A Bayesian perspective. Boca Raton, FL: CRC Press.

Edwards, D., \& Hamson, M. (2007). Guide to mathematical modelling. New York: Industrial Press.

Embretson, S. E. (2010). Measuring psychological constructs: Advances in model-based approaches. New York: American Psychological Association.

Epskamp, S., \& Fried, E. I. (2018). A tutorial on regularized partial correlation networks. Psychological Methods, 23(4), 617-634. https://doi.org/10.1037/met0000167

Epskamp, S., Rhemtulla, M., \& Borsboom, D. (2017). Generalized network psychometrics: Combining network and latent variable models. Psychometrika, 82(4), 904-927. https://doi.org/10.1007/s11336-017-9557-x

Erdfelder, E., Auer, T. S., Hilbig, B. E., Aßfalg, A., Moshagen, M., \& Nadarevic, L. (2009). Multinomial processing tree models: A review of the literature. Journal of Psychology, 217(3), 108-124. https://doi.org/10.1027/0044-3409.217.3.108

Faraway, J. J. (2016). Extending the linear model with R: Generalized linear, mixed effects and nonparametric regression models. Boca Raton, FL: CRC Press.

Fechner, G. T. (1860). Elemente der psychophysik [Elements of psychophysics]. Leipzig: Breitkopf \& Hartel. 
Ferraty, F., \& Vieu, P. (2006). Nonparametric functional data analysis: Theory and practice. New York: Springer.

Festinger, L. (1962). A theory of cognitive dissonance. Palo Alto, CA: Stanford University Press.

Furr, M. (2011). Scale construction and psychometrics for social and personality psychology. New York: Sage.

Golino, H. F., \& Demetriou, A. (2017). Estimating the dimensionality of intelligence like data using Exploratory Graph Analysis. Intelligence, 62, 54-70. https://doi.org/10.1016/j.intell.2017.02.007

Golino, H. F., \& Epskamp, S. (2017). Exploratory graph analysis: A new approach for estimating the number of dimensions in psychological research. PLOS One, 12(6), 1-26. https://doi.org/10.1371/journal.pone.0174035

Gould, S. J. (1996). The mismeasure of man. New York, NY: Norton.

Griffith, D. A., \& Akio, S. (1995). Trade-offs associated with normalizing constant computational simplifications for estimating spatial statistical models. Journal of Statistical Computation and Simulation, 51(2-4), 165-183.

https://doi.org/10.1080/00949659508811630

Hays, R. D., Morales, L. S., \& Reise, S. P. (2000). Item response theory and health outcomes measurement in the $21^{\text {st }}$ century. Medical Care, 38 (9 Suppl), 11281142.

Heene, M., Kyngdon, A., \& Sckopke, P. (2016). Detecting violations of unidimensionality by order-restricted inference methods. Frontiers in Applied Mathematics and Statistics, 2, 1-13. https://doi.org/10.3389/fams.2016.00003 
Hull, C. (1943). Principles of behavior. New York: Appleton-Century-Crofts.

Jones, L. V., \& Thissen, D. (2006). A history and overview of psychometrics. In C. R. Rao \& S. Sinharay (Eds.), Handbook of statistics: Volume 26 (pp. 1-27). New York: Elsevier.

Junker, B. W., \& Sijtsma, K. (2000). Latent and manifest monotonicity in item response models. Applied Psychological Measurement, 24(1), 65-81. https://doi.org/10.1177/01466216000241004

Kagan, J. (2005). A time for specificity. Journal of Personality Assessment, 85, 125 127. https://doi.org/10.1207/s15327752jpa8502_03

Kahneman, D., \& Tversky, A. (1979). Prospect theory: An analysis of decision under risk. Econometrica, 47(2), 262-292. https://doi.org/00129682(197903)47:2<263:PTAAOD>2.0.CO;2-3

Kanazawa, S. (1998). In defense of unrealistic assumptions. Sociological Theory, 16(2), 193-204. https://doi.org/10.1111/0735-2751.00050

Kim, E. J., Namkoong, K., Ku, T., \& Kim, S. J. (2008). The relationship between online game addiction and aggression, self-control and narcissistic personality traits. European Psychiatry, 23(3), 212-218. https://doi.org/10.1016/j.eurpsy.2007.10.010

Kish, L. (2004). Statistical design for research. New York: John Wiley \& Sons.

Knol, D. L., \& Berger, M. P. (1991). Empirical comparison between factor analysis and multidimensional item response models. Multivariate Behavioral Research, 26(3), 457-477. https://doi.org/10.1207/s15327906mbr2603_5 
Kruis, J., \& Maris, G. (2016). Three representations of the Ising model. Scientific Reports, 6, 1-11. https://doi.org/10.1038/srep34175

LaHuis, D. M., Blackmore, C. E., Bryant-Lees, K. B., \& Delgado, K. (2018). Applying Item Response Trees to personality data in the selection context. Organizational Research Methods, Online first, 1-12. https://doi.org/10.1177/1094428118780310

Lauritzen, S. L. (1996). Graphical models. Oxford: Clarendon Press.

Lee, M. D. (2011). How cognitive modeling can benefit from hierarchical Bayesian models. Journal of Mathematical Psychology, 55(1), 1-7. https://doi.org/10.1016/j.jmp.2010.08.013

Lee, M. D., \& Wagenmakers, E. J. (2014). Bayesian cognitive modeling: A practical course. Cambridge: Cambridge University Press.

Levine, M. V. (2003). Dimension in latent variable models. Journal of Mathematical Psychology, 47(4), 450-466. https://doi.org/10.1016/S0022-2496(03)00032-4

Lewandowsky, S., \& Farrell, S. (2010). Computational modeling in cognition: Principles and practice. New York: Sage.

Lord, F. M. (1953). An application of confidence intervals and of maximum likelihood to the estimation of an examinee's ability. Psychometrika, 18(1), 57-76. https://doi.org/10.1007/BF02289028

Manning, C. D., Manning, C. D., \& Schütze, H. (1999). Foundations of statistical natural language processing. Cambridge: MIT Press.

Marsman, M., Borsboom, D., Kruis, J., Epskamp, S., van Bork, R., Waldorp, L. J., van der Maas, \& Maris, G. (2018). An introduction to network psychometrics: 
Relating Ising network models to item response theory models. Multivariate Behavioral Research, 53(1), 15-35. https://doi.org/10.1080/00273171.2017.1379379

McDonald R. P. (2013). Test theory: A unified treatment. New York: Psychology Press.

McDonald, R. P. (1965). Difficulty factors and nonlinear factor analysis. British Journal of Mathematical and Statistical Psychology, 18(1), 11-23. https://doi.org/10.1111/j.2044-8317.1965.tb00690.x

Meijer, R. R. (1994). The number of Guttman errors as a simple and powerful person-fit statistic. Applied Psychological Measurement, 18(4), 311-314. https://doi.org/10.1177/014662169401800402

Mertens, D. M. (2014). Research and evaluation in education and psychology: Integrating diversity with quantitative, qualitative, and mixed methods. New York: Sage.

Michell, J. (1990). An introduction to the logic of psychological measurement. Hillsdale, NJ: Erlbaum.

Michell, J. (1997). Quantitative science and the definition of measurement in psychology. British Journal of Psychology, 88(3), 355-383. https://doi.org/10.1111/j.2044-8295.1997.tb02641.x

Michell, J. (2000). Normal science, pathological science and psychometrics. Theory \& Psychology, 10, 639-667. https://doi.org/10.1177/0959354300105004

Michell, J. (2005). The logic of measurement: A realist overview. Measurement, 38(4), 285-294. https://doi.org/10.1016/j.measurement.2005.09.004 
Michell, J. (2008). Conjoint measurement and the Rasch paradox: A response to Kyngdon. Theory \& Psychology, 18, 119-124. https://doi.org/10.1177/0959354307086926

Michell, J. (2017). On substandard substantive theory and axing axioms of measurement: A response to Humphry. Theory \& Psychology, 27(3), 419-425. https://doi.org/10.1177/0959354317706746

Miyazaki, K., \& Hoshino, T. (2009). A Bayesian semiparametric item response model with Dirichlet process priors. Psychometrika, 74(3), 375-393. https://doi.org/10.1007/s11336-008-9108-6

Mokken, R. J. (1971). A theory and procedure of scale analysis. The Hague: Mouton/Berlin: De Gruyter.

Mokken, R. J., \& Lewis, C. (1982). A nonparametric approach to the analysis of dichotomous item responses. Applied Psychological Measurement, 6, 417-430. https://doi.org/10.1177/014662168200600404

Myung, I. J., \& Pitt, M. A. (2001). Mathematical modeling. In J. Wixted (Ed.), Stevens' handbook of experimental psychology. Vol. 4: Methodology (pp. 429-459). New York: Wiley.

Nisbett, R. E., Aronson, J., Blair, C., Dickens, W., Flynn, J., Halpern, D. F., \& Turkheimer, E. (2012). Intelligence: New findings and theoretical developments. American Psychologist, 67(2), 130-159. https://doi.org/10.1037/a0026699

Nosofsky, R. M. (1986). Attention, similarity, and the identification-categorization relationship. Journal of Experimental Psychology: General, 115(1), 39-57. https://doi.org/10.1037/0096-3445.115.1.39

Nunnally, J. C. (1978). Psychometric theory. New York: McGraw-Hill. 
Price, D. J. S. (1986). Little science, big science... and beyond. New York: Columbia University Press.

Rasch, G. (1960). Studies in mathematical psychology: I. Probabilistic models for some intelligence and attainment tests. Copenhagen: Danish Educational Research Institute.

Reckase, M. (2009). Multidimensional item response theory. New York: Springer.

Reise, S. P., Ainsworth, A. T., \& Haviland, M. G. (2005). Item response theory: Fundamentals, applications, and promise in psychological research. Current Directions in Psychological Science, 14(2), 95-101. https://doi.org/10.1111/j.0963-7214.2005.00342.x

Rosenbaum, P. R. (1987). Comparing item characteristic curves. Psychometrika, 52(2), 217-233. https://doi.org/10.1007/BF02294236

Sandberg, J., \& Alvesson, M. (2011). Ways of constructing research questions: Gapspotting or problematization? Organization, 18(1), 23-44. https://doi.org/10.1177/1350508410372151

Shaughnessy, J. J., Zechmeister, E. B., \& Zechmeister, J. S. (2014). Research methods in psychology. New York: Alfred A. Knopf.

Shoenfield, J. R. (2018). Mathematical logic. Boca Raton, FL: CRC Press.

Sijtsma, K. (2012). Psychological measurement between physics and statistics. Theory \& Psychology, 22(6), 786-809. https://doi.org/10.1177/0959354312454353

Sijtsma, K., \& Molenaar, I. W. (2002). Introduction to nonparametric item response theory. New York: Sage. 
Stanislaw, H., \& Todorov, N. (1999). Calculation of signal detection theory measures. Behavior Research Methods, Instruments, \& Computers, 31(1), 137-149. https://doi.org/10.3758/BF03207704

Stanovich, K. E. (2012). How to think straight about psychology. New York: Pearson.

Stevens, S. S. (1946). On the theory of scales of measurement. Science, 103, 667-680. https://www.jstor.org/stable/1671815

Stout, W. (1987). A nonparametric approach for assessing latent trait unidimensionality. Psychometrika, 52(4), 589-617. https://doi.org/10.1007/BF02294821

Straat, J. H., Van der Ark, L. A., \& Sijtsma, K. (2013). Comparing optimization algorithms for item selection in Mokken scale analysis. Journal of Classification, 30(1), 75-99. https://doi.org/10.1007/s00357-013-9122-y

Thabane, L., Thomas, T., Ye, C., \& Paul, J. (2009). Posing the research question: Not so simple. Canadian Journal of Anesthesia, 56(1), 71-79. https://doi.org/10.1007/s12630-008-9007-4

Thompson, B. (2004). Exploratory and confirmatory factor analysis: Understanding concepts and applications. New York: American Psychological Association.

Townsend, J. T. (2008). Mathematical psychology: Prospects for the 21 st century: A guest editorial. Journal of Mathematical Psychology, 52(5), 269-280. https://doi.org/10.1016/j.jmp.2008.05.001

Trendler, G. (2009). Measurement theory, psychology and the revolution that cannot happen. Theory \& Psychology, 19(5), 579-599. https://doi.org/10.1177/0959354309341926 
Trendler, G. (2013). Measurement in psychology: A case of ignoramus et ignorabimus? A rejoinder. Theory \& Psychology, 23(5), 591-615. https://doi.org/10.1177/0959354313490451

Van der Linden, W. J., \& Hambleton, R. K. (2013). Handbook of modern item response theory. New York: Springer.

Van der Maas, H. L., Dolan, C. V., Grasman, R. P., Wicherts, J. M., Huizenga, H. M., \& Raijmakers, M. E. (2006). A dynamical model of general intelligence: The positive manifold of intelligence by mutualism. Psychological Review, 113(4), 842-861. https://doi.org/10.1037/0033-295X.113.4.842

Van der Maas, H., Kan, K. J., Marsman, M., \& Stevenson, C. E. (2017). Network models for cognitive development and intelligence. Journal of Intelligence, 5(2), 1-17. https://doi.org/10.3390/jintelligence5020016

Von Neumann, J., \& Morgenstern, O. (1944). Theory of games and economic behavior. Princeton: Princeton University Press.

Wang, C., Chang, H. H., \& Douglas, J. A. (2013). The linear transformation model with frailties for the analysis of item response times. British Journal of Mathematical and Statistical Psychology, 66(1), 144-168. https://doi.org/10.1111/j.20448317.2012.02045.x

Zhang, J., \& Stout, W. (1999). The theoretical DETECT index of dimensionality and its application to approximate simple structure. Psychometrika, 64(2), 213-249. https://doi.org/10.1007/BF02294536 